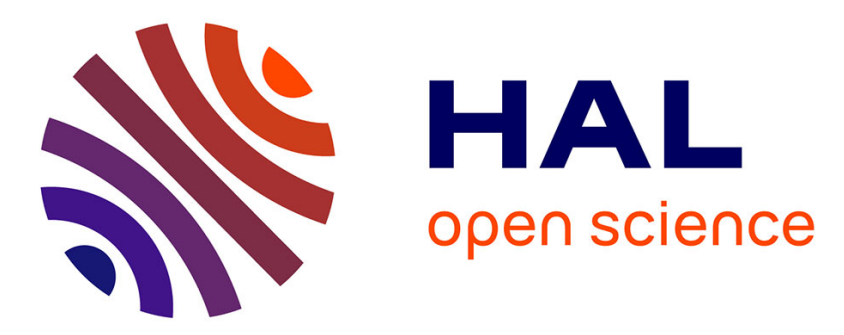

\title{
GEVREY INDEX THEOREM FOR THE INHOMOGENEOUS n-DIMENSIONAL HEAT EQUATION WITH A POWER-LAW NONLINEARITY AND VARIABLE COEFFICIENTS \\ Pascal Remy
}

\section{To cite this version:}

Pascal Remy. GEVREY INDEX THEOREM FOR THE INHOMOGENEOUS n-DIMENSIONAL HEAT EQUATION WITH A POWER-LAW NONLINEARITY AND VARIABLE COEFFICIENTS. 2019. hal-02117418

\author{
HAL Id: hal-02117418 \\ https://hal.science/hal-02117418
}

Preprint submitted on 2 May 2019

HAL is a multi-disciplinary open access archive for the deposit and dissemination of scientific research documents, whether they are published or not. The documents may come from teaching and research institutions in France or abroad, or from public or private research centers.
L'archive ouverte pluridisciplinaire HAL, est destinée au dépôt et à la diffusion de documents scientifiques de niveau recherche, publiés ou non, émanant des établissements d'enseignement et de recherche français ou étrangers, des laboratoires publics ou privés. 


\title{
GEVREY INDEX THEOREM FOR THE INHOMOGENEOUS $n$-DIMENSIONAL HEAT EQUATION WITH A POWER-LAW NONLINEARITY AND VARIABLE COEFFICIENTS
}

\author{
PASCAL REMY
}

\begin{abstract}
We are interested in the Gevrey properties of the formal power series solution in time of the inhomogeneous semilinear heat equation with a power-law nonlinearity in 1-dimensional time variable $t \in \mathbb{C}$ and $n$-dimensional spatial variable $x \in \mathbb{C}^{n}$ and with analytic initial condition and analytic coefficients at the origin $x=0$. We prove in particular that the inhomogeneity of the equation and the formal solution are together $s$-Gevrey for any $s \geqslant 1$. In the opposite case $s<1$, we show that the solution is 1-Gevrey at most while the inhomogeneity is $s$-Gevrey, and we give an explicit example in which the solution is $s^{\prime}$-Gevrey for no $s^{\prime}<1$.
\end{abstract}

\section{Setting the Problem}

For several years, various works have been done on the divergent solutions of some classes of linear partial differential equations or integro-differential equations in two variables or more, allowing thus to formulate many results on Gevrey properties, summability or multisummability (e.g. $[2,4-7,9,11,12,14,19,21,23,24,27-35,41$, $42,45-47,55,57])$.

In the case of the nonlinear partial differential equations, the situation is much more complicated. The existing results concern mainly Gevrey properties, especially the convergence (e.g. $[10,16,18,20,25,36-38,48-54])$, and there are very few results about the summation (see $[17,22,26,40,43]$ ).

In this article, we propose to investigate the Gevrey properties of the inhomogeneous semilinear heat equation

$$
\left\{\begin{array}{l}
\partial_{t} u-a(x) \Delta_{x} u-b(x) u^{m}=\tilde{f}(t, x) \\
u(0, x)=\varphi(x)
\end{array}\right.
$$

with a 1-dimensional time variable $t \in \mathbb{C}$ and a $n$-dimensional spatial variable $x:=\left(x_{1}, \ldots, x_{n}\right) \in \mathbb{C}^{n}$, where

- $\Delta_{x}:=\partial_{x_{1}}^{2}+\ldots+\partial_{x_{n}}^{2}$ is the Laplace operator;

- the coefficients $a(x)$ and $b(x)$ and the initial condition $\varphi(x)$ are analytic on a polydisc $\mathcal{D}_{\rho_{1}, \ldots, \rho_{n}}:=D_{\rho_{1}} \times \ldots \times D_{\rho_{n}}$ centered at the origin of $\mathbb{C}^{n}\left(D_{\rho}\right.$ denotes the disc with center $0 \in \mathbb{C}$ and radius $\rho>0$ );

- the degree $m$ of the power-law nonlinearity is an integer $\geqslant 2$;

2000 Mathematics Subject Classification. 35C10, 35K05, 35K55, 40B05.

Key words and phrases. Gevrey order, Heat equation, Inhomogeneous partial differential equation, Nonlinear partial differential equation, Formal power series, Divergent power series. 
- the inhomogeneity $\tilde{f}(t, x)$ is a formal power series in $t$ with analytic coefficients in $\mathcal{D}_{\rho_{1}, \ldots, \rho_{n}}$ (we denote by $\left.\tilde{f}(t, x) \in \mathcal{O}\left(\mathcal{D}_{\rho_{1}, \ldots, \rho_{n}}\right)[[t]]\right)$ which may be smooth, or not ${ }^{1}$.

Equation (1.1) arises in many physical, chemical, biological, and ecological problems involving diffusion and nonlinear growth such as heat and mass transfer, combustion theory, and spread theory of animal or plant populations. For example, if a chemical reaction generates heat at a rate depending on the temperature $u$, then $u$ satisfies equation eq. (1.1). In biological and ecological problems, the nonlinear term $u^{m}$ represents the growth of animal or plant population.

Let us now write the inhomogeneity $\tilde{f}(t, x)$ on the form

$$
\tilde{f}(t, x)=\sum_{j \geqslant 0} f_{j, *}(x) \frac{t^{j}}{j !}
$$

with $f_{j, *}(x) \in \mathcal{O}\left(\mathcal{D}_{\rho_{1}, \ldots, \rho_{n}}\right)$ for all $j \geqslant 0$. Then, it is clear that equation eq. (1.1) admits a unique formal series solution

$$
\widetilde{u}(t, x) \sum_{j \geqslant 0} u_{j, *}(x) \frac{t^{j}}{j !} \in \mathcal{O}\left(\mathcal{D}_{\rho_{1}, \ldots, \rho_{n}}\right)[[t]],
$$

where, for all $j \geqslant 0$, the coefficients $u_{j, *}(x) \in \mathcal{O}\left(\mathcal{D}_{\rho_{1}, \ldots, \rho_{n}}\right)$ are recursively determined from $u_{0, *}(x)=\varphi(x)$ by the relations

$$
\begin{aligned}
& u_{j+1, *}(x)=f_{j, *}(x)+a(x) \Delta_{x} u_{j, *}(x)+ \\
& \quad b(x) \sum_{k_{1}+\ldots+k_{m}=j} \frac{j !}{k_{1} ! \ldots k_{m} !} u_{k_{1}, *}(x) \ldots u_{k_{m}, *}(x) .
\end{aligned}
$$

An important particular case of equation eq. (1.1) is the case $a(x)=1, b(x)=0$, and $\tilde{f}(t, x)=0$, for which equation eq. (1.1) becomes the classical linear heat initial conditions problem

$$
\left\{\begin{array}{l}
\partial_{t} u=\Delta_{x} u \\
u(0, x)=\varphi(x)
\end{array}\right.
$$

In this case, it is well-known that the solution $\widetilde{u}(t, x)$ is generally divergent and generically 1 -Gevrey $[19,27]$. This Gevrey property was extended later to the nonconstant case $a(x) \neq 1[9]$ and to the inhomogeneous case $\tilde{f}(t, x) \neq 0[5,28]$. In the latter case, it was proved in particular that the solution $\widetilde{u}(t, x)$ and the inhomogeneity $\widetilde{f}(t, x)$ are together 1-Gevrey.

In the present paper, we consider the very general equation eq. (1.1), where no restrictive assumption is made on the coefficients $a(x)$ and $b(x)$, on the inhomogeneity $\tilde{f}(t, x)$ and on the initial condition $\varphi(x)$, except the assumption that $a(x)$, $b(x)$ and $\varphi(x)$ are analytic at the origin $x=0$ of $\mathbb{C}^{n}$ and the assumption that $\tilde{f}(t, x)$ is a formal power series in $t$ with analytic coefficients at $x=0^{2}$.

Let us mention here that a real variant of equation eq. (1.1) was already studied in the case $a(x)=1, b(x)=1$ and $\tilde{f}(t, x)=0$ by T. Gramchev and G. Lysik

\footnotetext{
${ }^{1}$ We denote $\tilde{f}$ with a tilde to emphasize the possible divergence of the series $\tilde{f}$.

${ }^{2}$ Thereby, our study includes in particular the case $b(x)=0$; hence, the linear case. Therefore, all the results stated in this article generalize the results already known for the classical heat equation.
} 
[10], then by G. Lysik and S. Michalik [20] by replacing the nonlinearity $u^{m}$ by a polynomial in $u$ with constant coefficients, and has shown that the solution $\widetilde{u}(t, x)$ is again 1 -Gevrey.

The organization of the paper is as follows. In section 2, we recall the definition and some properties about the $s$-Gevrey formal power series in $\mathcal{O}\left(\mathcal{D}_{\rho_{1}, \ldots, \rho_{n}}\right)[[t]]$ which are needed in the sequel. Section 3 is devoted to the main result of the article which states that the solution $\widetilde{u}(t, x)$ and the inhomogeneity $\widetilde{f}(t, x)$ are together $s$-Gevrey for any $s \geqslant 1$ (theorem 3.1), generalizing thus the results already known for the linear heat equation (see references just above). In the opposite case $s<1$, we show that $\widetilde{u}(t, x)$ is 1 -Gevrey at most while $\widetilde{f}(t, x)$ is $s$-Gevrey and an explicit example for which $\widetilde{u}(t, x)$ is $s^{\prime}$-Gevrey for no $s^{\prime}<1$ is displayed (proposition 3.2). A detailed proof of the main theorem 3.1 is developed in section 4 . This one is based on the Nagumo norms, a technique of majorant series and a fixed point procedure.

\section{Gevrey Formal Series}

Before stating our main result (see theorem 3.1 below), let us first recall for the convenience of the reader some definitions and basic properties about the Gevrey formal series in $\mathcal{O}\left(\mathcal{D}_{\rho_{1}, \ldots, \rho_{n}}\right)[[t]]$, which are needed in the sequel.

All along the article, we consider $t$ as the variable and $x$ as a parameter. Thereby, to define the notion of Gevrey classes of formal power series in $\mathcal{O}\left(\mathcal{D}_{\rho_{1}, \ldots, \rho_{n}}\right)[[t]]$, one extends the classical notion of Gevrey classes of elements in $\mathbb{C}[[t]]$ to families parametrized by $x$ in requiring similar conditions, the estimates being however uniform with respect to $x$. Doing that, any formal power series of $\mathcal{O}\left(\mathcal{D}_{\rho_{1}, \ldots, \rho_{n}}\right)[[t]]$ can be seen as a formal power series in $t$ with coefficients in a convenient Banach space defined as the space of functions that are holomorphic on a polydisc $\mathcal{D}_{\rho, \ldots, \rho}$ $\left(0<\rho \leqslant \min \rho_{\ell}\right)$ and continuous up to its boundary, equipped with the usual supremum norm. For a general study of series with coefficients in a Banach space, we refer for instance to [3].

In the sequel, we endow $\mathbb{C}^{n}$ with the maximum norm: for $x=\left(x_{1}, \ldots, x_{n}\right) \in \mathbb{C}^{n}$,

$$
\|x\|=\max _{\ell \in\{1, \ldots, n\}}\left|x_{\ell}\right| .
$$

Definition 2.1. Let $s \geqslant 0$ be. A formal series

$$
\widetilde{u}(t, x)=\sum_{j \geqslant 0} u_{j, *}(x) \frac{t^{j}}{j !} \in \mathcal{O}\left(\mathcal{D}_{\rho_{1}, \ldots, \rho_{n}}\right)[[t]]
$$

is said to be Gevrey of order $s$ (in short, s-Gevrey) if there exist three positive constants $0<\rho<\min \rho_{\ell}, C>0$ and $K>0$ such that the inequalities

$$
\sup _{\|x\| \leqslant \rho}\left|u_{j, *}(x)\right| \leqslant C K^{j} \Gamma(1+(s+1) j)
$$

hold for all $j \geqslant 0$.

In other words, definition 2.1 means that $\widetilde{u}(t, x)$ is $s$-Gevrey in $t$, uniformly in $x$ on a neighborhood of $x=(0, \ldots, 0) \in \mathbb{C}^{n}$.

We denote by $\mathcal{O}\left(\mathcal{D}_{\rho_{1}, \ldots, \rho_{n}}\right)[[t]]_{s}$ the set of all the formal series in $\mathcal{O}\left(\mathcal{D}_{\rho_{1}, \ldots, \rho_{n}}\right)[[t]]$ which are $s$-Gevrey. Observe that the set $\mathbb{C}\{t, x\}$ of germs of analytic functions at the origin of $\mathbb{C}^{n+1}$ coincides with the union $\bigcup_{\rho_{1}>0, \ldots, \rho_{n}>0} \mathcal{O}\left(\mathcal{D}_{\rho_{1}, \ldots, \rho_{n}}\right)[[t]]_{0}$; in particular, any element of $\mathcal{O}\left(\mathcal{D}_{\rho_{1}, \ldots, \rho_{n}}\right)[[t]]_{0}$ is convergent and $\mathbb{C}\{t, x\} \cap \mathcal{O}\left(\mathcal{D}_{\rho_{1}, \ldots, \rho_{n}}\right)[[t]]=$ 
$\mathcal{O}\left(\mathcal{D}_{\rho_{1}, \ldots, \rho_{n}}\right)[[t]]_{0}$. Observe also that the sets $\mathcal{O}\left(\mathcal{D}_{\rho_{1}, \ldots, \rho_{n}}\right)[[t]]_{s}$ are filtered as follows:

$$
\mathcal{O}\left(\mathcal{D}_{\rho_{1}, \ldots, \rho_{n}}\right)[[t]]_{0} \subset \mathcal{O}\left(\mathcal{D}_{\rho_{1}, \ldots, \rho_{n}}\right)[[t]]_{s} \subset \mathcal{O}\left(\mathcal{D}_{\rho_{1}, \ldots, \rho_{n}}\right)[[t]]_{s^{\prime}} \subset \mathcal{O}\left(\mathcal{D}_{\rho_{1}, \ldots, \rho_{n}}\right)[[t]]
$$

for all $s$ and $s^{\prime}$ satisfying $0<s<s^{\prime}<+\infty$.

Following proposition 2.2 specifies the algebraic structure of $\mathcal{O}\left(\mathcal{D}_{\rho_{1}, \ldots, \rho_{n}}\right)[[t]]_{s}$.

Proposition 2.2. Let $s \geqslant 0$. Then, the $\operatorname{set}\left(\mathcal{O}\left(\mathcal{D}_{\rho_{1}, \ldots, \rho_{n}}\right)[[t]]_{s}, \partial_{t}, \partial_{x_{1}}, \ldots, \partial_{x_{n}}\right)$ is a $\mathbb{C}$-differential algebra.

Proof. Since $\left(\mathcal{O}\left(\mathcal{D}_{\rho_{1}, \ldots, \rho_{n}}\right)[[t]], \partial_{t}, \partial_{x_{1}}, \ldots, \partial_{x_{n}}\right)$ is a $\mathbb{C}$-differential algebra, it is sufficient to prove that $\mathcal{O}\left(\mathcal{D}_{\rho_{1}, \ldots, \rho_{n}}\right)[[t]]_{s}$ is stable under multiplication and derivations.

The proof of the stability under the multiplication and the derivation $\partial_{t}$ is similar to the one already detailed in [45, Prop. 1] (see also [3, p. 64]) in the case $n=1$.

To prove the stability under the derivation $\partial_{x_{\ell}}$ with $\ell \in\{1, \ldots, n\}$, we proceed as follows. Let $\widetilde{u}(t, x) \in \mathcal{O}\left(\mathcal{D}_{\rho_{1}, \ldots, \rho_{n}}\right)[[t]]_{s}$ as in definition 2.1 and $\widetilde{w}(t, x)=\partial_{x_{\ell}} \widetilde{u}(t, x)$. For a given $0<\rho^{\prime}<\rho$, the Cauchy integral formula gives us, for all $j \geqslant 0$ and all $\|x\| \leqslant \rho^{\prime}$ :

$$
w_{j, *}(x)=\partial_{x_{\ell}} u_{j, *}(x)=\frac{1}{(2 i \pi)^{n}} \int_{\gamma(x)} \frac{u_{j, *}\left(x^{\prime}\right)}{\left(x_{\ell^{\prime}}-x_{\ell}\right)^{2} \prod_{\substack{k=1 \\ k \neq \ell}}^{n}\left(x_{k}^{\prime}-x_{k}\right)} d x^{\prime},
$$

where $\gamma(x):=\left\{x^{\prime}=\left(x_{1}^{\prime}, \ldots, x_{n}^{\prime}\right) \in \mathbb{C}^{n} ;\left|x_{k}^{\prime}-x_{k}\right|=\rho-\rho^{\prime}\right.$ for all $\left.k \in\{1, \ldots, n\}\right\}$. Hence, the inequalities

$$
\sup _{\|x\| \leqslant \rho^{\prime}}\left|w_{j, *}(x)\right| \leqslant C^{\prime} K^{j} \Gamma(1+(s+1) j) \quad \text { with } C^{\prime}=\frac{C}{\rho-\rho^{\prime}} \text { for all } j \geqslant 0 .
$$

Indeed, the definition of the path $\gamma(x)$ implies $\left\|x^{\prime}\right\| \leqslant \rho$. The proof is complete.

Observe that the stability under the derivation $\partial_{x_{\ell}}$ would not be guaranteed without the condition "there exist $0<\rho<\min \rho_{\ell} \ldots$ " in definition 2.1.

Observe also that a direct consequence of proposition 2.2 is the following.

Corollary 2.3. Suppose that the formal solution $\widetilde{u}(t, x)$ of equation eq. (1.1) is $s$-Gevrey for some $s \geqslant 0$. Then, the inhomogeneity $\tilde{f}(t, x)$ is $s$-Gevrey too.

In section 3 below, we are interested in the following question which ensues naturally from corollary 2.3 :

$$
\begin{gathered}
\text { "If } \tilde{f}(t, x) \text { is s-Gevrey for some } s \geqslant 0, \\
\text { is the formal solution } \widetilde{u}(t, x) \text { s-Gevrey too?" }
\end{gathered}
$$

As we shall prove, the response to this question depends on the value of the index $s$ and, more precisely, on the value of $s$ in relation to the critical value $s_{c}=1$.

\section{Gevrey Index Theorem}

In the case $s \geqslant 1$, the Gevrey Index Theorem below (see theorem 3.1) asserts that the converse of corollary 2.3 is true, providing thus a positive answer to Question eq. (Q). 
Theorem 3.1 (Gevrey Index Theorem). The formal solution $\widetilde{u}(t, x)$ of equation eq. (1.1) and the inhomogeneity $\tilde{f}(t, x)$ are together $s$-Gevrey for any $s \geqslant 1$.

On the other hand, in the opposite case $s<1$, the answer to Question eq. (Q) is generally negative (hence, the converse of corollary 2.3 is false). In fact, according to the filtration of the $s$-Gevrey spaces $\mathcal{O}\left(\mathcal{D}_{\rho_{1}, \ldots, \rho_{n}}\right)[[t]]_{s}$ (see section 2) and theorem 3.1, we can only be said that the formal solution $\widetilde{u}(t, x)$ of equation eq. (1.1) is generically 1-Gevrey at most when $\tilde{f}(t, x)$ is $s$-Gevrey with $s<1$. Proposition 3.2 below gives just us an example in which $\widetilde{u}(t, x)$ is $s^{\prime}$-Gevrey for no $s^{\prime}<1$.

Proposition 3.2 (A counter-example for $s<1$ ). Let $\varphi(x)$ be the analytic fonction on $\mathcal{D}:=\mathcal{D}_{1 / n, \ldots, 1 / n}$ defined by

$$
\varphi(x)=\frac{1}{1-x_{1}-\ldots-x_{n}} .
$$

Suppose that the inhomogeneity $\widetilde{f}(t, x)$ satisfies:

- $\tilde{f}(t, x)$ is $s$-Gevrey for some $s<1$,

- $\partial_{x}^{\alpha} f_{j, *}(0) \geqslant 0$ for all $\alpha \in \mathbb{N}^{n}$ and all $j \geqslant 0^{3}$.

Suppose also that the coefficients $a(x)$ and $b(x)$ are analytic on $\mathcal{D}$ and satisfy the inequalities $a(0)>0, \partial_{x}^{\alpha} a(0) \geqslant 0$ and $\partial_{x}^{\alpha} b(0) \geqslant 0$ for all $\alpha \in \mathbb{N}^{n}$.

Then, the formal solution $\widetilde{u}(t, x)$ of equation eq. (1.1) is exactly 1-Gevrey.

Proof. It is sufficient to prove that $\widetilde{u}(t, x)$ is $s^{\prime}$-Gevrey for no $s^{\prime}<1$.

Using the recurrence relations eq. (1.2), we first have

$$
u_{j, *}(x)=(n a(x))^{j}(2 j) !(\varphi(x))^{2 j+1}+\varphi(x) \operatorname{rem}_{j}(\varphi(x))
$$

for all $x \in \mathcal{D}$ and all $j \geqslant 0$, where $\operatorname{rem}_{j}(X)$ is a polynomial in $X$, the coefficients of which read on the form

$$
c \prod_{\substack{\alpha, \beta, \gamma \in \mathbb{N}^{n} \\ j^{\prime} \in\{0, \ldots, j\} \\ \text { finite }}}\left(\partial_{x}^{\alpha} a(x)\right)^{k_{\alpha}}\left(\partial_{x}^{\beta} b(x)\right)^{k_{\beta}}\left(\partial_{x}^{\gamma} f_{j^{\prime}, *}(x)\right)^{k_{\gamma, j^{\prime}}},
$$

with $c>0$ and $k_{\alpha}, k_{\beta}, k_{\gamma, j^{\prime}} \in \mathbb{N}$. In particular, for $x=0$, the assumptions on $a(x)$, $b(x)$ and the $f_{j^{\prime}, *}(x)$ 's lead us to the inequalities

$$
u_{j, *}(0) \geqslant(n a(0))^{j} \Gamma(1+2 j)>0 \text { for all } j \geqslant 0 .
$$

Let us now suppose that $\widetilde{u}(t, x)$ is $s^{\prime}$-Gevrey for some $s^{\prime}<1$. Then, definition 2.1 and eq. (3.1) imply the relations

$$
1<C\left(\frac{K}{n a(0)}\right)^{j} \frac{\Gamma\left(1+\left(s^{\prime}+1\right) j\right)}{\Gamma(1+2 j)}
$$

for all $j \geqslant 0$ and some convenient positive constants $C$ and $K$ independent of $j$. Proposition 3.2 follows since such inequalities are impossible. Indeed, applying the Stirling's Formula, we get

$$
C\left(\frac{K}{n a(0)}\right)^{j} \frac{\Gamma\left(1+\left(s^{\prime}+1\right) j\right)}{\Gamma(1+2 j)} \underset{j \rightarrow+\infty}{\sim} C \sqrt{\frac{s^{\prime}+1}{2}}\left(\frac{K\left(s^{\prime}+1\right)^{s^{\prime}+1} e^{1-s^{\prime}}}{4 n a(0) j^{1-s^{\prime}}}\right)^{j}
$$

which goes to 0 when $j$ tends to infinity. This ends the proof.

\footnotetext{
${ }^{3}$ As usual, we set $\partial_{x}^{\alpha}:=\partial_{x_{1}}^{\alpha_{1}} \ldots \partial_{x_{n}}^{\alpha_{n}}$ while $\alpha=\left(\alpha_{1}, \ldots, \alpha_{n}\right)$.
} 
Observe that theorem 3.1 and proposition 3.2 apply in particular in the case $b(x)=0$, that is in the case of the classical $n$-dimensional inhomogeneous heat initial conditions problem

$$
\left\{\begin{array}{l}
\partial_{t} u-a(x) \Delta_{x} u=\tilde{f}(t, x) \\
u(0, x)=\varphi(x)
\end{array}\right.
$$

generalizing thus the results already proved in $[5,9,19,27,28]$.

Section 4 below is devoted to the proof of the main theorem 3.1.

\section{Proof of theorem 3.1}

We have just to prove the converse of corollary 2.3 .

Let us fix $s \geqslant 1$ and let us suppose that the inhomogenity $\tilde{f}(t, x)$ is $s$-Gevrey. By assumption, its coefficients $f_{j, *}(x) \in \mathcal{O}\left(\mathcal{D}_{\rho_{1}, \ldots, \rho_{n}}\right)$ satisfy the following condition (see definition 2.1): there exist three positive constants $0<\rho<\min \rho_{\ell}, C>0$ and $K>0$ such that the inequalities

$$
\left|f_{j, *}(x)\right| \leqslant C K^{j} \Gamma(1+(s+1) j)
$$

hold for all $j \geqslant 0$ and all $\|x\| \leqslant \rho$.

We must prove that the coefficients $u_{j, *}(x) \in \mathcal{O}\left(\mathcal{D}_{\rho_{1}, \ldots, \rho_{n}}\right)$ of $\tilde{u}(t, x)$ satisfy similar inequalities. The approach we present below is analoguous to the ones already developed in [5, 45-47] in the framework of linear partial and integro-differential equations and is based on the Nagumo norms $[8,39,56]$ and on a technique of majorant series. However, our calculations appear to be much more complicated than in the linear case: the nonlinear term $u^{m}$ of equation eq. (1.1) generates indeed several new technical combinatorial situations.

Before starting the calculations, let us first recall for the convenience of the reader the definition of the Nagumo norms and some of their properties which are needed in the sequel.

\subsection{Nagumo Norms.}

Definition 4.1. Let $f \in \mathcal{O}\left(\mathcal{D}_{\rho_{1}, \ldots, \rho_{n}}\right), p \geqslant 0$ and $0<r<\min \rho_{\ell}$ be. Then, the Nagumo norm $\|f\|_{p, r}$ with indices $(p, r)$ of $f$ is defined by

$$
\|f\|_{p, r}:=\sup _{\|x\| \leqslant r}\left|f(x) d_{r}(x)^{p}\right|
$$

where $d_{r}(x)$ denotes the Euclidian distance $d_{r}(x):=r-\|x\|$.

Following proposition 4.2 gives us some properties of the Nagumo norms.

Proposition 4.2. Let $f, g \in \mathcal{O}\left(\mathcal{D}_{\rho_{1}, \ldots, \rho_{n}}\right), p, p^{\prime} \geqslant 0$ and $0<r<\min \rho_{\ell}$ be. Then,

(1) $\|\cdot\|_{p, r}$ is a norm on $\mathcal{O}\left(\mathcal{D}_{\rho_{1}, \ldots, \rho_{n}}\right)$.

(2) $|f(x)| \leqslant\|f\|_{p, r} d_{r}(x)^{-p}$ for all $\|x\|<r$.

(3) $\|f\|_{0, r}=\sup _{\|x\| \leqslant r}|f(x)|$ is the usual sup-norm on the polydisc $\mathcal{D}_{r, \ldots, r}$.

(4) $\|f g\|_{p+p^{\prime}, r} \leqslant\|f\|_{p, r}\|g\|_{p^{\prime}, r}$.

(5) $\left\|\partial_{x_{\ell}} f\right\|_{p+1, r} \leqslant e(p+1)\|f\|_{p, r}$ for all $\ell \in\{1, \ldots, n\}$. 
Proof. Properties 1-4 are straightforward and are left to the reader. To prove Property 5 , we proceed as follows. Let $\ell \in\{1, \ldots, n\}$ be, $x \in \mathbb{C}^{n}$ such that $\|x\|<r$ and $0<R<d_{r}(x)$. Using the Cauchy Integral Formula, we have

$$
\partial_{x_{\ell}} f(x)=\frac{1}{(2 i \pi)^{n}} \int_{\gamma(x)} \frac{f\left(x^{\prime}\right)}{\left(x_{\ell^{\prime}}-x_{\ell}\right)^{2} \prod_{\substack{k=1 \\ k \neq \ell}}^{n}\left(x_{k}^{\prime}-x_{k}\right)} d x^{\prime},
$$

where $\gamma(x):=\left\{x^{\prime}=\left(x_{1}^{\prime}, \ldots, x_{n}^{\prime}\right) \in \mathbb{C}^{n} ;\left|x_{k}^{\prime}-x_{k}\right|=R\right.$ for all $\left.k \in\{1, \ldots, n\}\right\}$. Since

$$
x^{\prime} \in \gamma(x) \Rightarrow\left\|x^{\prime}\right\|<r,
$$

we can apply Property 2 of proposition 4.2 ; hence, the inequalities

$$
\left|\partial_{x_{\ell}} f(x)\right| \leqslant \frac{1}{R} \max _{x^{\prime} \in \gamma(x)}\left|f\left(x^{\prime}\right)\right| \leqslant \frac{1}{R}\|f\|_{p, r} \max _{x^{\prime} \in \gamma(x)} d_{r}\left(x^{\prime}\right)^{-p}=\frac{1}{R}\|f\|_{p, r}\left(d_{r}(x)-R\right)^{-p} .
$$

Observe that the last equality stems from the relations

$$
d_{r}\left(x^{\prime}\right)=r-\left\|x^{\prime}\right\|=r-\left\|x+x^{\prime}-x\right\| \geqslant d_{r}(x)-\left\|x^{\prime}-x\right\|=d_{r}(x)-R>0 .
$$

When $p=0$, the choice $R=\frac{d_{r}(x)}{e}$ implies the inequality

$$
\left|\partial_{x_{\ell}} f(x)\right| \leqslant e\|f\|_{0, r} d_{r}(x)^{-1}
$$

hence, the inequality

$$
\left|\partial_{x_{\ell}} f(x)\right| d_{r}(x) \leqslant e\|f\|_{0, r} .
$$

When $p>0$, the choice $R=\frac{d_{r}(x)}{p+1}$ and the relations

$$
\left(1-\frac{1}{p+1}\right)^{-p}=\left(1+\frac{1}{p}\right)^{p}<e
$$

brings us to the inequalities

$$
\left|\partial_{x_{\ell}} f(x)\right| \leqslant\|f\|_{p, r} d_{r}(x)^{-p-1}(p+1)\left(1-\frac{1}{p+1}\right)^{-p} \leqslant e(p+1)\|f\|_{p, r} d_{r}(x)^{-p-1}
$$

and then to the inequality

$$
\left|\partial_{x_{\ell}} f(x)\right| d_{r}(x)^{p+1} \leqslant e(p+1)\|f\|_{p, r} .
$$

Property 5 follows since inequalities eqs. (4.2) and (4.3) are still valid when $\|x\|=r$. This achieves the proof of proposition 4.2.

Remark 4.3. Inequalities $4-5$ of proposition 4.2 are the most important properties. Observe besides that the same index $r$ occurs on their both sides, allowing thus to get estimates for the product $f g$ in terms of $f$ and $g$ and for the derivatives $\partial_{x_{\ell}} f$ for any $\ell \in\{1, \ldots, n\}$ in terms of $f$ without having to shrink the polydisc $\mathcal{D}_{r, \ldots, r}$.

Let us now turn to the proof of theorem 3.1. 
4.2. Some Inequalities. From recurrence relations eq. (1.2), we first derive the following identities for all $j \geqslant 0$ :

$$
\begin{aligned}
& \frac{u_{j+1, *}(x)}{\Gamma(1+(s+1)(j+1))}= \frac{f_{j, *}(x)}{\Gamma(1+(s+1)(j+1))}+\frac{a(x) \Delta_{x} u_{j, *}(x)}{\Gamma(1+(s+1)(j+1))}+ \\
& b(x) \sum_{k_{1}+\ldots+k_{m}=j} \frac{j !}{k_{1} ! \ldots k_{m} !} \frac{u_{k_{1}, *}(x) \ldots u_{k_{m}, *}(x)}{\Gamma(1+(s+1)(j+1))},
\end{aligned}
$$

with the initial condition $u_{0, *}(x)=\varphi(x)$. Let us now define the constant $A>0$ by

$$
A:=\left\|u_{0, *}\right\|_{0, \rho}=\|\varphi\|_{0, \rho}
$$

and let us apply the Nagumo norm of indices $((s+1)(j+1), \rho)$ to relations eq. (4.4).

From Property 1 of proposition 4.2, we first obtain:

$$
\begin{aligned}
\frac{\left\|u_{j+1, *}\right\|_{(s+1)(j+1), \rho}}{\Gamma(1+(s+1)(j+1))} \leqslant \frac{\left\|f_{j, *}\right\|_{(s+1)(j+1), \rho}}{\Gamma(1+(s+1)(j+1))}+\frac{\left\|a \Delta_{x} u_{j, *}\right\|_{(s+1)(j+1), \rho}}{\Gamma(1+(s+1)(j+1))}+ \\
\sum_{k_{1}+\ldots+k_{m}=j} \frac{j !}{k_{1} ! \ldots k_{m} !} \frac{\left\|b u_{k_{1}, *} \ldots u_{k_{m}, *}\right\|_{(s+1)(j+1), \rho}}{\Gamma(1+(s+1)(j+1))} .
\end{aligned}
$$

Then, Properties $4-5$ of proposition 4.2 imply the inequalities

$$
\begin{aligned}
& \frac{\left\|u_{j+1, *}\right\|_{(s+1)(j+1), \rho}}{\Gamma(1+(s+1)(j+1))} \leqslant \frac{\left\|f_{j, *}\right\|_{(s+1)(j+1), \rho}}{\Gamma(1+(s+1)(j+1))}+\alpha_{j, s} \frac{\left\|u_{j, *}\right\|_{(s+1) j, \rho}}{\Gamma(1+(s+1) j)}+ \\
&\|b\|_{s+1, \rho} \sum_{k_{1}+\ldots+k_{m}=j} \beta_{j, k_{1}, \ldots, k_{m}, s} \frac{\left\|u_{k_{1}, *}\right\|_{(s+1) k_{1}, \rho}}{\Gamma\left(1+(s+1) k_{1}\right)} \cdots \frac{\left\|u_{k_{m}, *}\right\|_{(s+1) k_{m}, \rho}}{\Gamma\left(1+(s+1) k_{m}\right)},
\end{aligned}
$$

where the constants $\alpha_{j, s}$ and $\beta_{k_{1}, \ldots, k_{m}, j}$ are defined by

$$
\begin{aligned}
& \alpha_{j, s}:=\frac{n e^{2}((s+1) j+2)((s+1) j+1)\|a\|_{s-1, \rho} \Gamma(1+(s+1) j)}{\Gamma(1+(s+1)(j+1))}, \\
& \beta_{j, k_{1}, \ldots, k_{m}, s}:=\frac{j !}{k_{1} ! \ldots k_{m} !} \frac{\Gamma\left(1+(s+1) k_{1}\right) \ldots \Gamma\left(1+(s+1) k_{m}\right)}{\Gamma(1+(s+1)(j+1))} .
\end{aligned}
$$

Observe that all the norms, especially the norm $\|a\|_{s-1, \rho}$, are well-defined since $s \geqslant 1$.

Following propositions 4.4 and 4.5 allow us to bound the constants $\alpha_{j, s}$ and $\beta_{j, k_{1}, \ldots, k_{m}, s}$.

Proposition 4.4. Let $j \geqslant 0$ be. Then,

$$
\frac{((s+1) j+2)((s+1) j+1) \Gamma(1+(s+1) j)}{\Gamma(1+(s+1)(j+1))} \leqslant 1 .
$$

Proof. Applying the recurrence formula $\Gamma(z+1)=z \Gamma(z)$ twice, we first have

$$
((s+1) j+2)((s+1) j+1) \Gamma(1+(s+1) j)=\Gamma(1+(s+1) j+2) .
$$

Inequality eq. (4.6) follows then from the relations

$$
1+(s+1)(j+1)=1+(s+1) j+s+1 \geqslant 1+(s+1) j+2 \geqslant 2
$$

(we have indeed $s \geqslant 1$ ) and from the increase of the Gamma function on $[2 ;+\infty[$. 
Proposition 4.5. Let $j \geqslant 0$ be and $k_{1}, \ldots, k_{m} \in \mathbb{N}$ such that $k_{1}+\ldots+k_{m}=j$. Then,

$$
\frac{\Gamma\left(1+(s+1) k_{1}\right) \ldots \Gamma\left(1+(s+1) k_{m}\right)}{\Gamma(1+(s+1)(j+1))} \leqslant \frac{k_{1} ! \ldots k_{m} !}{j !} .
$$

Proof. First of all, let us write the left-hand side of inequality eq. (4.7) on the form

$$
\frac{\Gamma\left(1+(s+1) k_{1}\right) \ldots \Gamma\left(1+(s+1) k_{m}\right)}{\Gamma(1+(s+1)(j+1))}=\frac{\Gamma(1+(s+1) j)}{\Gamma(1+(s+1)(j+1))} Q_{j, k_{1}, \ldots, k_{m}}(s+1),
$$

where $Q$ is the function defined on $[0 ;+\infty[$ by

$$
Q_{j, k_{1}, \ldots, k_{m}}(q)=\frac{\Gamma\left(1+k_{1} q\right) \ldots \Gamma\left(1+k_{m} q\right)}{\Gamma(1+j q)} .
$$

Since proposition 4.4 implies

$$
\frac{\Gamma(1+(s+1) j)}{\Gamma(1+(s+1)(j+1))} \leqslant \frac{((s+1) j+2)((s+1) j+1) \Gamma(1+(s+1) j)}{\Gamma(1+(s+1)(j+1))} \leqslant 1
$$

it is sufficient to prove that

$$
Q_{j, k_{1}, \ldots, k_{m}}(s+1) \leqslant \frac{k_{1} ! \ldots k_{m} !}{j !} .
$$

To this end, let us study the variations of the function $Q_{j, k_{1}, \ldots, k_{m}}$. This latter is derivable on $[0 ;+\infty[$ and, for all $q \geqslant 0$, we have

$$
Q_{j, k_{1}, \ldots, k_{m}}^{\prime}(q)=Q_{j, k_{1}, \ldots, k_{m}}(q)\left(\sum_{\ell=1}^{m} k_{\ell} \psi\left(1+k_{\ell} q\right)-j \psi(1+j q)\right),
$$

where $\psi:=\Gamma^{\prime} / \Gamma$ is the Digamma function. Thereby, applying the classical relation (see $[1$, p. 259] for instance)

$$
\psi(1+q)=-\gamma+\sum_{h=1}^{+\infty} \frac{q}{h(h+q)} \quad, q \geqslant 0, \gamma:=\text { the Euler's constant }
$$

we get

$$
Q_{j, k_{1}, \ldots, k_{m}}^{\prime}(q)=q Q_{j, k_{1}, \ldots, k_{m}}(q) \sum_{h=1}^{+\infty}\left(\sum_{\ell=1}^{m} \frac{k_{\ell}^{2}}{h\left(h+k_{\ell} q\right)}-\frac{j^{2}}{h(h+j q)}\right) .
$$

Next, lemma 4.6 below shows us that $Q_{j, k_{1}, \ldots, k_{m}}^{\prime}(q) \leqslant 0$ for all $q \geqslant 0$ and, consequently, the function $Q_{j, k_{1}, \ldots, k_{m}}$ is decreasing on $[0 ;+\infty[$. Hence,

$$
Q_{j, k_{1}, \ldots, k_{m}}(q) \leqslant Q_{j, k_{1}, \ldots, k_{m}}(1)=\frac{\Gamma\left(1+k_{1}\right) \ldots \Gamma\left(1+k_{m}\right)}{\Gamma(1+j)}=\frac{k_{1} ! \ldots k_{m} !}{j !}
$$

for all $q \geqslant 1$ and inequality eq. (4.9) stems from the relation $s+1 \geqslant 2$. This ends the proof.

Lemma 4.6. Let $q \geqslant 0$ and $h \geqslant 1$ be. Then, the inequality

$$
\sum_{\ell=1}^{m} \frac{k_{\ell}^{2}}{h+k_{\ell} q} \leqslant \frac{\left(\sum_{\ell=1}^{m} k_{\ell}\right)^{2}}{h+\left(\sum_{\ell=1}^{m} k_{\ell}\right) q}
$$

holds for all $m \geqslant 2$ and all $k_{1}, \ldots, k_{m} \geqslant 0$. 
Proof. We proceed by induction on $m$. For $m=2$, we clearly have

$$
\frac{k_{1}^{2}}{h+k_{1} q}+\frac{k_{2}^{2}}{h+k_{2} q}-\frac{\left(k_{1}+k_{2}\right)^{2}}{h+\left(k_{1}+k 2\right) q}=\frac{-k_{1} k_{2} h\left(k_{1} q+k_{2} q+2 h\right)}{\left(h+k_{1} q\right)\left(h+k_{2} q\right)\left(h+\left(k_{1}+k_{2}\right) q\right)} \leqslant 0 .
$$

Let us now suppose that inequality eq. (4.10) is true for all $k \in\{2, \ldots, m\}$ for a certain $m \geqslant 2$. Then, the successive relations

$$
\begin{aligned}
\sum_{\ell=1}^{m+1} \frac{k_{\ell}^{2}}{h+k_{\ell} q} \leqslant & \frac{\left(\sum_{\ell=1}^{m} k_{\ell}\right)^{2}}{h+\left(\sum_{\ell=1}^{m} k_{\ell}\right) q}+\frac{k_{m+1}^{2}}{h+k_{m+1} q} \\
\leqslant & \frac{\left(\sum_{\ell=1}^{m} k_{\ell}+k_{m+1}\right)^{2}}{h+\left(\sum_{\ell=1}^{m} k_{\ell}+k_{m+1}\right) q}=\frac{\left(\sum_{\ell=1}^{m+1} k_{\ell}\right)^{2}}{h+\left(\sum_{\ell=1}^{m+1} k_{\ell}\right) q}
\end{aligned}
$$

hold for any $k_{1}, \ldots, k_{m+1} \geqslant 0$, which achieves the proof.

Let us now apply propositions 4.4 and 4.5 : we get

$$
\alpha_{j, s} \leqslant n e^{2}\|a\|_{s-1, \rho} \text { and } \beta_{j, k_{1}, \ldots, k_{m}, s} \leqslant 1 .
$$

Hence, the following inequalities

$$
\begin{aligned}
& \frac{\left\|u_{j+1, *}\right\|_{(s+1)(j+1), \rho}}{\Gamma(1+(s+1)(j+1))} \leqslant \frac{\left\|f_{j, *}\right\|_{(s+1)(j+1), \rho}}{\Gamma(1+(s+1)(j+1))}+ \\
& n e^{2}\|a\|_{s-1, \rho} \frac{\left\|u_{j, *}\right\|_{(s+1) j, \rho}}{\Gamma(1+(s+1) j)}+ \\
&\|b\|_{s+1, \rho} \sum_{k_{1}+\ldots+k_{m}=j} \frac{\left\|u_{k_{1}, *}\right\|_{(s+1) k_{1}, \rho}}{\Gamma\left(1+(s+1) k_{1}\right)} \cdots \frac{\left\|u_{k_{m}, *}\right\|_{(s+1) k_{m}, \rho}}{\Gamma\left(1+(s+1) k_{m}\right)}
\end{aligned}
$$

hold for all $j \geqslant 0$.

We now shall bound the Nagumo norms $\left\|u_{j, *}\right\|_{(s+1) j, \rho}$ for any $j$. To do that, we shall proceed similarly as in [5,45-47] by using a technique of majorant series. However, as we shall see, the calculations are much more complicated.

4.3. A Majorant Series. Let us consider the formal series $v(X)=\sum_{j \geqslant 0} v_{j} X^{j}$, where the coefficients $v_{j}$ are recursively determined from $v_{0}=A$ (see identity eq. (4.5) for the definition of $A$ ) by the relations

$$
v_{j+1}=\alpha v_{j}+g_{j}+\beta \sum_{k_{1}+\ldots+k_{m}=j} v_{k_{1}} \ldots v_{k_{m}}
$$

with $\alpha:=n e^{2}\|a\|_{s-1, \rho}, \beta:=\|b\|_{s+1, \rho}$ and

$$
g_{j}:=\frac{\left\|f_{j, *}\right\|_{(s+1)(j+1), \rho}}{\Gamma(1+(s+1)(j+1))} .
$$


By construction, we have

$$
0 \leqslant \frac{\left\|u_{j, *}\right\|_{(s+1) j, \rho}}{\Gamma(1+(s+1) j)} \leqslant v_{j}
$$

for all $j \geqslant 0$ (proceed by induction on $j$ ). Following proposition 4.7 allows us to bound the $v_{j}$ 's.

Proposition 4.7. The formal series $v(X)$ is convergent. In particular, there exist two positive constants $C^{\prime}, K^{\prime}>0$ such that $v_{j} \leqslant C^{\prime} K^{\prime j}$ for all $j \geqslant 0$.

Proof. It is sufficient to prove the convergence of $v(X)$.

First of all, let us observe that $v(X)$ is the unique formal power series in $X$ solution of the functional equation

$$
(1-\alpha X) v(X)=\beta X(v(X))^{m}+h(X),
$$

where

$$
h(X):=A+X \sum_{j \geqslant 0} g_{j} X^{j}
$$

is a convergent power series with nonnegative coefficients. Indeed, according to the assumption on the $f_{j}$ 's (see inequality eq. (4.1) at the beginning of section 4 ) and inequality eq. (4.8), we have

$$
0 \leqslant g_{j} \leqslant \frac{C K^{j} \Gamma(1+(s+1) j) \rho^{(s+1)(j+1)}}{\Gamma(1+(s+1)(j+1))} \leqslant C \rho^{s+1}\left(K \rho^{s+1}\right)^{j} .
$$

We denote in the sequel by $r_{h}>0$ the radius of convergence of $h$.

Next, we proceed through a fixed point method as follows. Let us set

$$
V(X)=\sum_{i \geqslant 0} V_{i}(X)
$$

and let us choose the solution of equation eq. (4.14) given by the system

$$
\left\{\begin{array}{l}
(1-\alpha X) V_{0}(X)=h(X) \\
(1-\alpha X) V_{i+1}(X)=\beta X \sum_{k_{1}+\ldots+k_{m}=i} V_{k_{1}}(X) \ldots V_{k_{m}}(X) \quad \text { for } i \geqslant 0 .
\end{array}\right.
$$

By induction on $i \geqslant 0$, we easily check that

$$
V_{i}(X)=\frac{C_{i, m} \beta^{i} X^{i}(h(X))^{i(m-1)+1}}{(1-\alpha X)^{i m+1}},
$$

where the $C_{i, m}$ 's are the positive constants recursively determined from $C_{0, m}:=1$ by the relations

$$
C_{i+1, m}=\sum_{k_{1}+\ldots+k_{m}=i} C_{k_{1}, m} \ldots C_{k_{m}, m} .
$$

Thereby, all the $V_{i}$ 's are analytic functions on the disc with center $0 \in \mathbb{C}$ and radius $\min \left(1 / \alpha, r_{h}\right)$. Moreover, identities eq. (4.15) tell us that $V_{i}(X)$ is of order $X^{i}$ for all $i \geqslant 0$. Consequently, the series $V(X)$ makes sense as a formal power series in $X$ and we get $V(X)=v(X)$ by unicity.

We are left to prove the convergence of $V(X)$. To do that, let us choose $0<$ $r<\min \left(1 / \alpha, r_{h}\right)$. By definition, the constants $C_{i, m}$ 's are the generalized Catalan 
numbers of order $m^{4}$ and we have

$$
C_{i, m}=\frac{1}{(m-1) i+1}\left(\begin{array}{c}
i m \\
i
\end{array}\right) \leqslant 2^{i m}
$$

for all $i \geqslant 0$ (see $[13,15,44]$ for instance). On the other hand, the convergent series $h(X)$ defines an increasing function on $[0, r]$. Therefore, identities eq. (4.15) imply the inequalities

$$
\left|V_{i}(X)\right| \leqslant \frac{h(r)}{1-\alpha r}\left(\frac{2^{m} \beta(h(r))^{m-1}}{(1-\alpha r)^{m}}|X|\right)^{i}
$$

for all $i \geqslant 0$ and all $|X| \leqslant r$. Consequently, the series $V(X)$ is normally convergent on any disc with center $0 \in \mathbb{C}$ and radius

$$
0<r^{\prime}<\min \left(r, \frac{(1-\alpha r)^{m}}{2^{m} \beta(h(r))^{m-1}}\right) .
$$

This proves the analyticity of $V(X)$ at 0 and achieves then the proof of proposition 4.7.

According to relations eq. (4.13), proposition 4.7 allows us to also bound the Nagumo norms $\left\|u_{j, *}\right\|_{(s+1) j, \rho}$.

Corollary 4.8. Let $C^{\prime}, K^{\prime}>0$ be as in proposition 4.7. Then, the inequalities

$$
\left\|u_{j, *}\right\|_{(s+1) j, \rho} \leqslant C^{\prime} K^{\prime j} \Gamma(1+(s+1) j)
$$

hold for all $j \geqslant 0$.

We are now able to conclude the proof of theorem 3.1.

4.4. Conclusion. We must prove on the sup-norm of the $u_{j}(x)$ estimates similar to the ones on the norms $\left\|u_{j, *}\right\|_{(s+1) j, \rho}$ (see corollary 4.8). To this end, we proceed by shrinking the closed polydisc $\|x\| \leqslant \rho$. Let $0<\rho^{\prime}<\rho$. Then, for all $j \geqslant 0$ and all $\|x\| \leqslant \rho^{\prime}$, we have

$$
\left|u_{j, *}(x)\right|=\left|u_{j, *}(x) d_{\rho}(x)^{(s+1) j} \frac{1}{d_{\rho}(x)^{(s+1) j}}\right| \leqslant \frac{\left|u_{j, *}(x) d_{\rho}(x)^{(s+1) j}\right|}{\left(\rho-\rho^{\prime}\right)^{(s+1) j}} \leqslant \frac{\left\|u_{j, *}\right\|_{(s+1) j, \rho}}{\left(\rho-\rho^{\prime}\right)^{(s+1) j}}
$$

and, consequently,

$$
\sup _{\|x\| \leqslant \rho^{\prime}}\left|u_{j, *}(x)\right| \leqslant C^{\prime}\left(\frac{K^{\prime}}{\left(\rho-\rho^{\prime}\right)^{s+1}}\right)^{j} \Gamma(1+(s+1) j)
$$

by applying corollary 4.8. This ends the proof of theorem 3.1 .

\footnotetext{
${ }^{4}$ These numbers were named in honor of the Belgian mathematician Eugène Charles Catalan (1814-1894). They appear in many probabilist, graphs and combinatorial problems. For example, they can be seen as the number of $m$-ary trees with $i$ source-nodes, or as the number of ways of associating $i$ applications of a given $m$-ary operation, or as the number of ways of subdividing a convex polygon into $i$ disjoint $(m+1)$-gons by means of non-intersecting diagonals. They also appear in theoretical computers through the generalized Dyck words. See for instance [13] and the references inside.
} 


\section{REFERENCES}

[1] M. Abramowitz and I. A. Stegun. Handbook of mathematical functions with formulas, graphs, and mathematical tables. Dover Publications, INC., New-York, 1965.

[2] W. Balser. Divergent solutions of the heat equation: on an article of Lutz, Miyake and Schäfke. Pacific J. Math., 188(1):53-63, 1999.

[3] W. Balser. Formal power series and linear systems of meromorphic ordinary differential equations. Universitext. Springer-Verlag, New-York, 2000.

[4] W. Balser. Multisummability of formal power series solutions of partial differential equations with constant coefficients. J. Differential Equations, 201(1):63-74, 2004.

[5] W. Balser and M. Loday-Richaud. Summability of solutions of the heat equation with inhomogeneous thermal conductivity in two variables. Adv. Dyn. Syst. Appl., 4(2):159-177, 2009.

[6] W. Balser and M. Miyake. Summability of formal solutions of certain partial differential equations. Acta Sci. Math. (Szeged), 65(3-4):543-551, 1999.

[7] W. Balser and M. Yoshino. Gevrey order of formal power series solutions of inhomogeneous partial differential equations with constant coefficients. Funkcial. Ekvac., 53:411-434, 2010.

[8] M. Canalis-Durand, J.-P. Ramis, R. Schäfke, and Y. Sibuya. Gevrey solutions of singularly perturbed differential equations. J. Reine Angew. Math., 518:95-129, 2000.

[9] O. Costin, H. Park, and Y. Takei. Borel summability of the heat equation with variable coefficients. J. Differential Equations, 252(4):3076-3092, 2012.

[10] T. Gramchev and G. Lysik. Uniform analytic-Gevrey regularity of solutions to a semilinear heat equation. Banach Center Publ., 81:213-226, 2008.

[11] M. Hibino. Borel summability of divergence solutions for singular first-order partial differential equations with variable coefficients. I \& II. J. Differential Equations, 227(2):499-563, 2006.

[12] M. Hibino. On the summability of divergent power series solutions for certain first-order linear PDEs. Opuscula Math., 35(5):595-624, 2015.

[13] P. Hilton and J. Pedersen. Catalan numbers, their generalization, and their uses. Math. Intelligencer, 13(2):64-75, 1991.

[14] K. Ichinobe. On $k$-summability of formal solutions for a class of partial differential operators with time dependent coefficients. J. Differential Equations, 257(8):3048-3070, 2014.

[15] D. A. Klarner. Correspondences between plane trees and binary sequences. J. Combinatorial Theory, 9:401-411, 1970.

[16] A. Lastra and S. Malek. On parametric Gevrey asymptotics for some nonlinear initial value Cauchy problems. J. Differential Equations, 259:5220-5270, 2015.

[17] A. Lastra and S. Malek. On parametric multisummable formal solutions to some nonlinear initial value Cauchy problems. Adv. Differ. Equ., 2015:200, 2015.

[18] A. Lastra, S. Malek, and J. Sanz. On Gevrey solutions of threefold singular nonlinear partial differential equations. J. Differential Equations, 255:3205-3232, 2013.

[19] D. A. Lutz, M. Miyake, and R. Schäfke. On the Borel summability of divergent solutions of the heat equation. Nagoya Math. J., 154:1-29, 1999.

[20] G. Lysik and S. Michalik. Formal solutions of semilinear heat equations. J. Math. Anal. Appl., 341:372-385, 2008.

[21] S. Malek. On the summability of formal solutions of linear partial differential equations. $J$. Dyn. Control Syst., 11(3):389-403, 2005.

[22] S. Malek. On the summability of formal solutions of nonlinear partial differential equations with shrinkings. J. Dyn. Control Syst., 13(1):1-13, 2007.

[23] S. Malek. On the Stokes phenomenon for holomorphic solutions of integrodifferential equations with irregular singularity. J. Dyn. Control Syst., 14(3):371-408, 2008.

[24] S. Malek. On Gevrey functions solutions of partial differential equations with fuchsian and irregular singularities. J. Dyn. Control Syst., 15(2):277-305, 2009.

[25] S. Malek. On Gevrey asymptotic for some nonlinear integro-differential equations. J. Dyn. Control Syst., 16(3):377-406, 2010.

[26] S. Malek. On the summability of formal solutions for doubly singular nonlinear partial differential equations. J. Dyn. Control Syst., 18(1):45-82, 2012.

[27] S. Michalik. Summability of divergent solutions of the $n$-dimensional heat equation. J. Differential Equations, 229:353-366, 2006. 
[28] S. Michalik. Summability of formal solutions to the $n$-dimensional inhomogeneous heat equation. J. Math. Anal. Appl., 347:323-332, 2008.

[29] S. Michalik. On the multisummability of divergent solutions of linear partial differential equations with constant coefficients. J. Differential Equations, 249:551-570, 2010.

[30] S. Michalik. Summability and fractional linear partial differential equations. J. Dyn. Control Syst., 16(4):557-584, 2010.

[31] S. Michalik. Multisummability of formal solutions of inhomogeneous linear partial differential equations with constant coefficients. J. Dyn. Control Syst., 18(1):103-133, 2012.

[32] S. Michalik. Corrigendum to "On the multisummability of divergent solutions of linear partial differential equations with constant coefficients" [j. differential equations 249 (3) (2010) 551570]. J. Differential Equations, 255:2400-2401, 2013.

[33] M. Miyake. Newton polygons and formal Gevrey indices in the Cauchy-Goursat-Fuchs type equations. J. Math. Soc. Japan, 43(2):305-330, 1991.

[34] M. Miyake. Borel summability of divergent solutions of the Cauchy problem to nonKovaleskian equations. In Partial differential equations and their applications (Wuhan, 1999), pages 225-239. World Sci. Publ., River Edge, NJ, 1999.

[35] M. Miyake and Y. Hashimoto. Newton polygons and Gevrey indices for linear partial differential operators. Nagoya Math. J., 128:15-47, 1992.

[36] M. Miyake and A. Shirai. Convergence of formal solutions of first order singular nonlinear partial differential equations in the complex domain. Ann. Polon. Math., 74:215-228, 2000.

[37] M. Miyake and A. Shirai. Structure of formal solutions of nonlinear first order singular partial differential equations in complex domain. Funkcial. Ekvac., 48:113-136, 2005.

[38] M. Miyake and A. Shirai. Two proofs for the convergence of formal solutions of singular first order nonlinear partial differential equations in complex domain. Surikaiseki Kenkyujo Kokyuroku Bessatsu, Kyoto Unviversity, B37:137-151, 2013.

[39] M. Nagumo. Über das Anfangswertproblem partieller Differentialgleichungen. Jap. J. Math., 18:41-47, 1942.

[40] S. Ouchi. Genuine solutions and formal solutions with Gevrey type estimates of nonlinear partial differential equations. J. Math. Sci. Univ. Tokyo, 2:375-417, 1995.

[41] S. Ouchi. Multisummability of formal solutions of some linear partial differential equations. J. Differential Equations, 185(2):513-549, 2002.

[42] S. Ouchi. Borel summability of formal solutions of some first order singular partial differential equations and normal forms of vector fields. J. Math. Soc. Japan, 57(2):415-460, 2005.

[43] M. E. Pliś and B. Ziemian. Borel resummation of formal solutions to nonlinear Laplace equations in 2 variables. Ann. Polon. Math., 67(1):31-41, 1997.

[44] G. Pólya and G. Szegö. Aufgaben und Lehrsätze aus der Analysis, Vol. I, volume 125. Springer-Verlag, Berlin, Göttingen, Heidelberg, 1954.

[45] P. Remy. Gevrey order and summability of formal series solutions of some classes of inhomogeneous linear partial differential equations with variable coefficients. J. Dyn. Control Syst., 22(4):693-711, 2016.

[46] P. Remy. Gevrey order and summability of formal series solutions of certain classes of inhomogeneous linear integro-differential equations with variable coefficients. J. Dyn. Control Syst., 23(4):853-878, 2017.

[47] P. Remy. Gevrey properties and summability of formal power series solutions of some inhomogeneous linear Cauchy-Goursat problems. J. Dyn. Control Syst., in press.

[48] A. Shirai. Maillet type theorem for nonlinear partial differential equations and newton polygons. J. Math. Soc. Japan, 53:565-587, 2001.

[49] A. Shirai. Convergence of formal solutions of singular first order nonlinear partial differential equations of totally characteristic type. Funkcial. Ekvac., 45:187-208, 2002.

[50] A. Shirai. A maillet type theorem for first order singular nonlinear partial differential equations. Publ. RIMS. Kyoto Univ., 39:275-296, 2003.

[51] A. Shirai. Maillet type theorem for singular first order nonlinear partial differential equations of totally characteristic type. Surikaiseki Kenkyujo Kokyuroku, Kyoto University, 1431:94$106,2005$.

[52] A. Shirai. Alternative proof for the convergence or formal solutions of singular first order nonlinear partial differential equations. Journal of the School of Education, Sugiyama Jogakuen University, 1:91-102, 2008. 
GEVREY INDEX THEOREM FOR INHOMOGENEOUS SEMILINEAR HEAT EQUATION 15

[53] A. Shirai. Gevrey order of formal solutions of singular first order nonlinear partial differential equations of totally characteristic type. Journal of the School of Education, Sugiyama Jogakuen University, 6:159-172, 2013.

[54] A. Shirai. Maillet type theorem for singular first order nonlinear partial differential equations of totally characteristic type, part ii. Opuscula Math., 35(5):689-712, 2015.

[55] H. Tahara and H. Yamazawa. Multisummability of formal solutions to the Cauchy problem for some linear partial differential equations. J. Differential Equations, 255(10):3592-3637, 2013.

[56] W. Walter. An elementary proof of the Cauchy-Kowalevsky theorem. Amer. Math. Monthly, 92(2):115-126, 1985.

[57] A. Yonemura. Newton polygons and formal Gevrey classes. Publ. Res. Inst. Math. Sci., 26:197-204, 1990.

Laboratoire de Mathématiques de Versailles, Université de Versailles Saint-Quentin, 45 aVenue des Etats-Unis, 78035 Versailles cedex, France

Email address: pascal.remy@uvsq.fr ; pascal.remy.maths@gmail.com 